\title{
Blurred Lines
}

Emmanuel Villermaux, IRPHE, Aix-Marseille Université, France

When lit from behind through a comb grid, a water jet is hardly discerned as its smooth surface leaves the projected grid unaltered. Increasing the flowrate causes turbulent hieratic motions to set in, which distort and corrugate the jet. Gridlines caustics are more distorted for higher flowrates, revealing ever smaller surface corrugations, varying on lengthscales smaller than the jet diameter. Eventually, turbulent fluctuations are intense enough to overcome surface tension confinement, and the jet fragments.

Drops formed this way result from a trade-off between disaggregation, and cohesion forces. The pictures are also a tribute to Harold Edgerton, whose first Moment of Vision, in 1932, was a turbulent jet from a faucet.

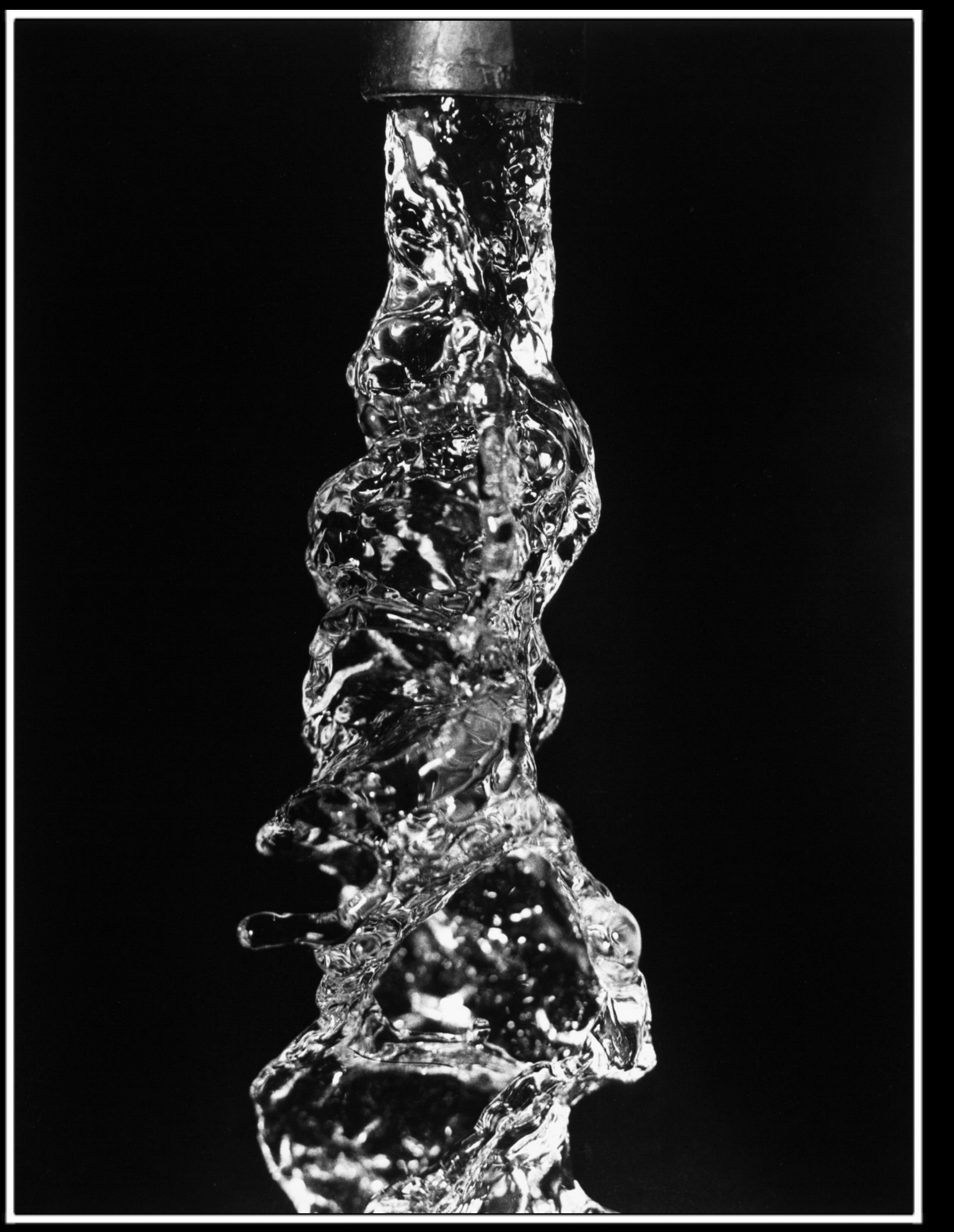

Harold Edgerton (1932)
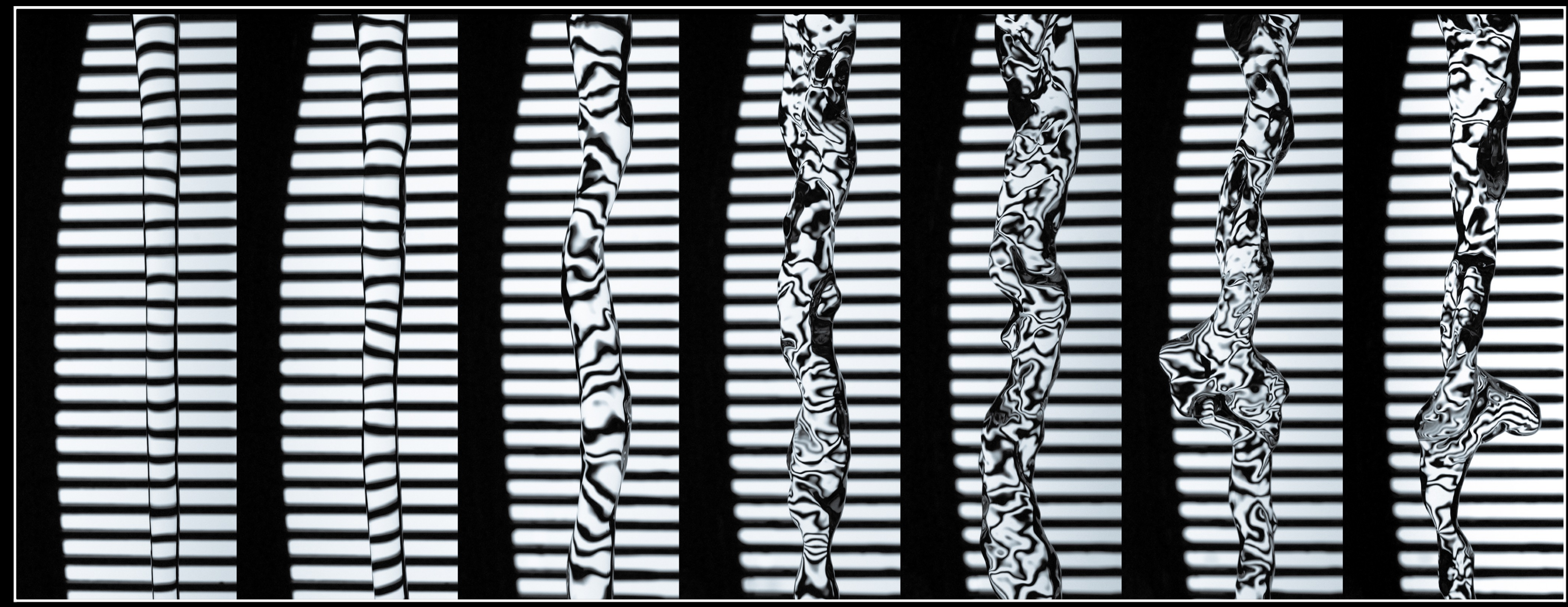